\title{
UM ALGORITMO BRANCH AND CUT PARA O PROBLEMA DE ROTEIRIZAÇÃO E ESTOQUES COM MÚLTIPLOS DEPÓSITOS E ENTREGAS FRACIONADAS
}

\author{
Cleder Marcos Schenekemberg \\ GTAO - Grupo de Tecnologia Aplicada à Otimização/UFPR \\ cledercms@hotmail.com \\ Thiago André Guimarães \\ GTAO - Grupo de Tecnologia Aplicada à Otimização/UFPR \\ thiagoandre@ufpr.br \\ Cassius Tadeu Scarpin \\ GTAO - Grupo de Tecnologia Aplicada à Otimização/UFPR \\ cassiusts@gmail.com
}

RESUMO

Neste trabalho resolvemos um Problema de Roteirização e Estoques com Múltiplos Depósitos e Entregas Fracionadas (Multi Depot Inventory Routing Problem with Split Deliveries - MDIRPSD). O Problema emerge no paradigma do sistemas de estoque gerenciado pelo fornecedor, quando as entregas são realizadas a partir de múltiplos depósitos e os clientes podem ser atendidos múltiplas vezes em um mesmo período. O MDIRPSD incorpora as decisões de suprimento e distribuição de forma simultânea e foi recentemente proposto na literatura. Nós apresentamos uma nova formulação matemática, propomos uma nova política de estoque e incorporamos os custos de estocagem dos clientes na otimização do problema. Também projetamos e implementamos um método exato que obteve resultados superiores ao branch-and-cut existente na literatura. Por fim, reportamos resultados comparativos entre as duas políticas de estoque consideradas.

Palavra-chave: Estoque Gerenciado pelo Fornecedor; Múltiplos Depósitos; Entregas Fracionadas; Método Exato.

\begin{abstract}
In this paper we solve a Multi Depot Inventory Routing Problem with Split Deliveries (MDIRPSD). The problem emerges under the vendor-managed inventory systems paradigm, when deliveries are made from multiple depots and the clients can be served several times over the same period. The MDIRPSD integrates supply and distribution decisions and has recently been proposed in the literature. We present a new mathematical formulation, taking into account inventory holding costs and also propose a new inventory policy. We have also designed and implemented a new branch-and-cut that overcome the similar approach in the literature. Finally we report comparative results between the two inventory policies considered.
\end{abstract}

Keywords: Vendor-managed inventory; Multi-depot; Split deliveries; Branch-and-cut. 


\section{INTRODUÇÃO}

Nas últimas décadas, a escalada competitiva global e a consequente redução das margens de lucro vem ensejando maior coordenação dos processos e atores no âmbito da cadeia de suprimentos. Ao mesmo tempo, o advento da indústria 4.0 e a disponibilização dados em tempo real, possibilitam uma agenda cada vez mais colaborativa nas atividades logísticas, com especial ênfase no elo fornecedor-cliente. Neste sentido, paradigmas que requerem a troca de informações e que esbarravam nos obstáculos tecnológicos de anos atrás, vem assumindo um protagonismo cada vez mais transversal. Não apenas pela redução nos custos dos sistemas de troca eletrônica de dados, mas também na robusta minimização de erros e incertezas que esses sistemas possibilitam, em comparação com técnicas indiretas de quantificação das demandas.

Os sistemas Vendor Managed Inventory (VMI) ou Estoque Gerenciado pelo Fornecedor, configuram uma prática gerencial colaborativa entre fornecedor e cliente, com inúmeros relatos de sucesso em diferentes segmentos (ver [1]). Sob um sistema VMI, o fornecedor controla o estoque do cliente, decidindo quando atendê-lo e quanto entregar por ocasião de um atendimento. A dinâmica dos estoques é diretamente dependente da demanda do cliente, que pode ser estimada por métodos de previsão, ou observada de forma precisa, através dos supracitados sistemas de troca eletrônica de dados. Segundo [2], a prática VMI é mutuamente benéfica, pois os clientes não precisam dispender recursos para controlar seus estoques e emitir pedidos de ressuprimento, enquanto os fornecedores ganham pela maior coordenação das atividades logísticas, particularmente na composição dos roteiros de entrega.

Pelo ângulo operacional, a implementação de um sistema $V M I$ implica na resolução de um problema de otimização combinatorial complexo, denominado Inventory-Routing Problem (IRP) ou Problema de Roteirização e Estoques. O IRP integra, em um mesmo arcabouço, o problema de gerenciamento de estoques e o problema de roteamento de veículos com múltiplos períodos. Nesta abordagem conjunta, compete ao fornecedor decidir: quando servir um cliente, quanto entregar a cada visita realizada e como definir as rotas de entrega. Tais decisões devem garantir que o cliente tenha sua demanda plenamente atendida, seja pelos estoques mantidos e/ou pelas quantidades recebidas.

Embora a literatura sobre o IRP reporte diversas variantes (ver [3]), a estrutura logística mais comum é bastante simplificada, envolvendo apenas um fornecedor, que opera a partir de um único depósito, e múltiplos clientes, com a proibição de entregas fracionadas [4. Todavia, em um contexto mais realístico, os clientes estão dispersos em uma ampla área urbana e com elevada densidade de tráfego. Ademais, o fornecedor geralmente dispõe de uma rede de depósitos e uma frota de veículos, o que eleva consideravelmente o número de opções distintas de servir a um cliente. Por esses aspectos, a possibilidade de entregas fracionadas se adere ao melhor uso dos recursos físicos do fornecedor (frota de veículos e múltiplos depósitos), ao mesmo tempo em que gerencia com mais eficiência as questões de tráfego e de logística urbana. Essas características definem um IRP com múltiplos depósitos e entregas fracionadas, a qual denominamos de Multi Depot Inventory Routing Problem with Split Deliveries (MDIRPSD), e que recentemente foi proposta por [5]. No trabalho, os autores introduzem o MDIRPSD e desenvolvem um algoritmo exato do tipo branch-and-cut, além de uma matheurística para resolver instâncias de maior porte. Ademais, o problema abordado não contempla o custo de estoque nos clientes, prática muito comum no IRP, (ver [6, 7, 8, 4]), e os autores consideram apenas a política de estoque Maximum Level $(M L)$, quando o fornecedor está livre para quantificar a entrega ao cliente, limitada à capacidade de estoque no período. 
Neste artigo, ampliamos o estudo do MDIRPSD e reformulamos o problema, a fim de incorporar o custo de estoque nos clientes nas decisões do fornecedor. Outrossim, introduzimos a política de estoque Order-up to Level (OU) no âmbito do MDIRPSD. A política $O U$ foi proposta por 9] para o IRP clássico e é frequentemente abordada por estudos na área [3]. Esta política exige que a quantidade entregue ao cliente complete a capacidade disponível de estoque, reduzindo o número de atendimentos ao longo do horizonte de planejamento. Por fim, propomos um novo algoritmo branch-and-cut para resolver o MDIRPSD de forma exata, considerando as políticas de estoque $M L$ e $O U$. Para a versão simplificada do MDIRPSD (política $M L$ e sem custo de estoque nos clientes), reportamos uma análise comparativa entre o algoritmo que propomos neste trabalho com método apresentado por [5]. Já para a extensão do MDIRPSD com custos de estoque, comparamos também as políticas $M L$ e $O U$ no âmbito do custo logístico global.

O restante do artigo está estruturado como segue. Na seção 2 definimos formalmente o MDIRPSD com custos de estoque nos clientes e apresentamos uma formulação para a política $O U$ com entregas fracionadas. Na seção 3 propomos o algoritmo branch-and-cut, enquanto a seção 4 reporta os resultados dos experimentos computacionais realizados. A seção 5 tece as conclusões do trabalho.

\section{DEFINIÇÃO DO PROBLEMA E MODELAGEM MATEMÁTICA}

O MDIRPSD é definido sobre um grafo incompleto e não direcionado $G=(\mathcal{V}, \mathcal{E})$, onde o conjunto de vértices $\mathcal{V}$ representa a união do conjunto de Plantas (ou depósitos) $\mathcal{P}$ e Clientes $\mathcal{C}$, enquanto o conjunto $\mathcal{E}$ são as arestas do grafo. Um custo não-negativo de transporte $c_{u v}$ está associado a cada aresta $(u, v) \in \mathcal{E}$, onde $\mathcal{E}=\{(u, v): u, v \in \mathcal{V} \wedge u, v \oplus \mathcal{P}, u<v\}$.

O horizonte de planejamento é definido pelo conjunto $\mathcal{T}$ com $p$ períodos. Uma frota homogênea de veículos, definida pelo conjunto $\mathcal{K}$ e com capacidade $Q$ por veículo, está disponível para realizar as entregas aos clientes. Cada veículo $k \in \mathcal{K}$ pode ser designado para uma planta $j, j \in \mathcal{P}$, em cada período $t, t \in \mathcal{T}$. Assume-se, sem perda de generalidade, que a capacidade de produção e estoque em cada planta $j$ é ilimitada.

Cada cliente $l, l \in \mathcal{C}$, tem uma demanda determinística não nula, $d_{l}^{t}$ a cada período $t$, $\operatorname{com} d_{l}^{0}=0$. A capacidade máxima de estoque e dada por $U_{l}$, sendo constante ao longo de $\mathcal{T}$, enquanto $I_{l}^{0}$ define o nível inicial de estoque, conhecido a priori. Por fim, cada cliente $l$ incorre em um custo de estoque $h_{l}$ por unidade estocada por período. O nível de estoque de cada cliente $l$, ao final de cada período $t, t \in \mathcal{T} \cup p+1$, é dado por $I_{l}^{t}$ e este não pode ser negativo.

Dois tipos de entregas fracionadas são permitidas a um cliente $l$ no mesmo período $t$ : quando dois ou mais veículos de uma mesma planta $j$ atendem ao referido cliente, bem como quando este mesmo cliente é servido por dois ou mais veículos de plantas distintas. No entanto, a rota de um veículo $k$ deve terminar na mesma planta $j$ de origem.

O objetivo do MDIRPSD é minimizar o custo logístico total, dado pelos componentes de estoque e transporte, determinando, para cada período $t$ :

- quando, quanto e a partir de quais plantas cada cliente deve ser atendido;

- quantos veículos designar a cada planta em cada período;

- como combinar as entregas dos clientes em cada período e em cada veículo; 
Com relação às variáveis de decisão, cada planta $j$ deve determinar a quantidade $q_{j l}^{k t}$ entregue ao cliente $l$, com o veículo $k$ no período $t$. O conjunto restante das variáveis é definido a seguir:

- $Y_{j l}^{k t}=1$ se o veículo $k$ designado à planta $j$ visita o vértice $l$ no período $t$, onde $l \in \mathcal{V}, 0$ caso contrário;

- $y_{u v}^{k j t}=1$ se o veículo $k$ designado à planta $j$ viaja do vértice $u$ ao vértice $v$ no período $t, \operatorname{com}(u, v) \in \mathcal{E}, u<v$ e $u, v \oplus \mathcal{P}, 0$ caso contrário;

Um adaptação da variável $Y_{j j}^{k t}=1$, anui o controle de saída do veículo $k$ da planta $j$ no período $t$. Finalmente, o MDIRPSD é formulado por (1)-(14).

$$
\min \sum_{t \in \mathcal{T} \cup\{p+1\}} \sum_{l \in \mathcal{C}} h_{l} I_{l}^{t}+\sum_{t \in \mathcal{T}} \sum_{j \in \mathcal{P}} \sum_{k \in \mathcal{K}} \sum_{(u, v) \in \mathcal{E}} c_{u v} y_{u v}^{k j t}
$$

subject to

$$
\begin{array}{rl}
I_{l}^{t}=I_{l}^{t-1}+\sum_{j \in \mathcal{P}} \sum_{k \in \mathcal{K}} q_{j l}^{k, t-1}-d_{l}^{t} & l \in \mathcal{C}, t \in \mathcal{T} \cup\{p+1\} \\
I_{l}^{t} \leq U_{l} & l \in \mathcal{C}, t \in \mathcal{T} \cup\{p+1\} \\
I_{l}^{t}+\sum_{j \in \mathcal{P}} \sum_{k \in \mathcal{K}} q_{j l}^{k t} \leq U_{l} & l \in \mathcal{C}, t \in \mathcal{T} \\
q_{j l}^{k t} \leq U_{l} Y_{j l}^{k t} & l \in \mathcal{C}, j \in \mathcal{P}, k \in \mathcal{K}, t \in \mathcal{T} \\
\sum_{l \in \mathcal{C}} q_{j l}^{k t} \leq Q Y_{j j}^{k t} & j \in \mathcal{P}, k \in \mathcal{K}, t \in \mathcal{T} \\
\sum_{j \in \mathcal{P}} Y_{j j}^{k t} \leq 1 & k \in \mathcal{K}, t \in \mathcal{T} \\
\sum_{u \in \mathcal{V}} y_{u l}^{k j t}+\sum_{u \in \mathcal{V}} y_{l u}^{k j t}=2 Y_{j l}^{k t} & l \in \mathcal{V}, j \in \mathcal{P}, k \in \mathcal{K}, t \in \mathcal{T} \\
\sum_{l \in S} \sum_{u \in S} y_{l u}^{k j t} \leq \sum_{l \in S} Y_{j l}^{k t}-Y_{j m}^{k t} & S \subseteq \mathcal{C},|S| \geq 2, m \in S, j \in \mathcal{P}, k \in \mathcal{K}, t \in \mathcal{T} \\
I_{l}^{t} \geq 0 & l \in \mathcal{C}, t \in \mathcal{T} \cup\{p+1\} \\
q_{j l}^{k t} \geq 0 & j \in \mathcal{P}, l \in \mathcal{C}, k \in \mathcal{K}, t \in \mathcal{T} \\
Y_{j l}^{k t} \in\{0,1\} & j \in \mathcal{P}, l \in \mathcal{V}, k \in \mathcal{K}, t \in \mathcal{T} \\
y_{j v}^{k j t} \in\{0,1,2\} & v \in \mathcal{C}, j \in \mathcal{P}, k \in \mathcal{K}, t \in \mathcal{T} \\
y_{u v}^{k j t} \in\{0,1\} & u, v \in \mathcal{C}, j \in \mathcal{P}, k \in \mathcal{K}, t \in \mathcal{T}
\end{array}
$$

A função objetivo 1 minimiza os custos totais, dado pela soma dos custos de estoque e transporte. As restrições 2 garantem a conservação de fluxo, enquanto 3 limitam os níveis de estoques nos clientes. A política $M L$ é formulada pelas restrições 4 Já as restrições 5 conectam a quantidade entregue pela planta $j$ ao cliente $l$ com o veículo $k$ se esse cliente for visitado pela tripla $(j, k, t)$. As restrições 6 impedem que a capacidade de um veículo $k$ seja excedida ao atender um conjunto de clientes, enquanto 7 garantem que cada veículo $k$ seja designado a uma única planta $j$ em cada período $t$. As restrições 8 e 9 estabelecem a conectividade dos vértices e proíbem a formação de sub rotas, respectivamente. O domínio das variáveis de decisão é dado pelas restricões $10-14$. 
Destacamos que modelo matemático (1) - (14) é suficientemente flexível para representar o problema proposto por [5]. Para isso, basta definir $h_{l}=0, l \in \mathcal{C}$. Adicionalmente, albergamos a quebra de simetria nas variáveis binárias que representam as decisões de rota ao modelar $y_{j v}^{k j t}=\{0,1,2\}$, reduzindo a um único arco as decisões de entregas diretas.

Em nosso melhor conhecimento, a literatura do IRP não reporta nenhuma formulação para a política $O U$ quando as entregas aos clientes podem ser fracionadas. De maneira a cobrir essa lacuna, definimos uma variável binária adicional $Z_{l}^{t}$, que assume o valor 1 quando o cliente $l$ é visitado ao menos uma vez no período $t$, e 0 caso contrário. As restrições $15-17$ asseguram a política $O U$ para o $M D I R P S D$, onde $M=|\mathcal{P}||\mathcal{K}|$ é um $B I G$ $M$.

$$
\begin{array}{rlrl}
\sum_{j \in \mathcal{P}} \sum_{k \in \mathcal{K}} Y_{j l}^{k t} \leq M Z_{l}^{t} & l \in \mathcal{C}, t \in \mathcal{T} \\
I_{l}^{t}+\sum_{j \in \mathcal{P}} \sum_{k \in \mathcal{K}} q_{j l}^{k t} \geq U_{l} Z_{l}^{t} & l \in \mathcal{C}, t \in \mathcal{T} \\
Z_{l}^{t} \in\{0,1\} & l \in \mathcal{C}, t \in \mathcal{T}
\end{array}
$$

De maneira a fortalecer os limitantes duais, nós apresentamos um conjunto de desigualdades válidas, propostas por [10, 11], para a variante clássica do IRP.

$$
\begin{array}{cl}
y_{j l}^{k j t} \leq 2 Y_{j l}^{k t} & j \in \mathcal{P}, l \in \mathcal{C}, k \in \mathcal{K}, t \in \mathcal{T} \\
y_{l u}^{k j t} \leq Y_{j l}^{k t} & j \in \mathcal{P}, l, u \in \mathcal{C}, l<u, k \in \mathcal{K}, t \in \mathcal{T} \\
y_{l u}^{k j t} \leq Y_{j u}^{k t} & j \in \mathcal{P}, l, u \in \mathcal{C}, l<u, k \in \mathcal{K}, t \in \mathcal{T} \\
Y_{j l}^{k t} \leq Y_{j j}^{k t} & j \in \mathcal{P}, l \in \mathcal{C}, k \in \mathcal{K}, t \in \mathcal{T}
\end{array}
$$

As desigualdades 18 elevam os limitantes inferiores do modelo, quando um cliente $l$ é atendido por uma entrega direta pelo veículo $k$ designado à planta $j$ no período $t$. Analogamente, 19 e 20 representam o caso de múltiplos clientes servidos em uma mesma rota. Por fim, as desigualdades 21 asseguram que o cliente $l$ poderá ser atendido pelo veículo $k$ que parte da planta $j$ no período $t$, somente se esse veículo for utilizado.

Adaptamos as restrições de quebra de simetria apresentadas por [11] para o IRP com múltiplos depósitos em 22, que garantem que um veículo de índice $k$ só será utilizado por uma planta $j$, quando o veículo de índice $k-1$ já estiver em uso por alguma planta. 


$$
\sum_{j \in \mathcal{P}} Y_{j j}^{k t} \leq \sum_{j \in \mathcal{P}} Y_{j j}^{k-1, t} \quad t \in \mathcal{T}, k \in \mathcal{K}, k>1
$$

Finalmente em 23, consideramos as desigualdades propostas por [4, que calculam o menor intervalo para o atendimento de um cliente, quando a estrutura logística é formada por múltiplos depósitos.

$$
\sum_{j \in \mathcal{P}} \sum_{k \in \mathcal{K}} \sum_{t=t_{1}}^{t_{2}} Y_{j l}^{k t} \geq\left\lceil\frac{\sum_{t=t_{1}}^{t_{2}} d_{l}^{t}-U_{l}}{\min \left\{Q, U_{l}\right\}}\right\rceil \quad l \in \mathcal{C}, t_{1}, t_{2} \in \mathcal{T}, t_{2}>t_{1}
$$

\section{ALGORITMO BRANCH-AND-CUT}

Por conta da sua complexidade combinatorial, o número de subconjuntos necessários para gerar todas as restrições de eliminação de subrotas (RES) em 9 é demasiadamente grande, fazendo com que o processo enumerativo pleno seja impraticável. Para superar essa limitação, essas restrições precisam ser geradas e adicionadas ao longo da otimização do problema. Nós delineamos uma abordagem exata para resolver o modelo apresentado na seção 2, onde as RES são adicionadas à arvore de busca, sempre que sub rotas forem identificadas na solução corrente. Esta técnica é conhecida como Branch-and-Cut $(B \mathscr{E} C)$, e combina a clássica metodologia Branch-and-Bound (BEBB) com algoritmos de planos de corte.

Durante a execução do algoritmo $B \mathscr{G} B$, a etapa de branching ou ramificação é a mais importante. Sempre que existirem variáveis de domínio inteiro com valores fracionários na solução corrente, uma ramificação deve ser imposta. Após uma criteriosa seleção, a variável fracionária escolhida $x=a$, limitar-se-à $x \geq\lceil a\rceil$ e $x \leq\lfloor a\rfloor$. Para o MDIRPSD, priorizamos a ramificação das variáveis de saída de um veículo da planta $Y_{j j}^{k t}$, seguido das variáveis de atendimento de cliente $Y_{j l}^{k t}$ e por último, as variáveis de rotas $y_{u v}^{k j t}$.

No início do processo de busca, todas as desigualdades válidas são geradas e adicionadas ao nó raiz da árvore do $B \mathscr{E} B$. A cada nó resolvido pelo método $B \mathscr{E} B$, um algoritmo de busca por RES violadas é então aplicado. Para essa finalidade nós utilizamos o pacote CVRPSEP, desenvolvido e disponibilizado por [12], que, ao identificar um RES violada, gera um conjunto de cortes e desigualdades válidas, as quais adaptamos para o MDIRPSD. Neste ponto, um novo subproblema é gerado pela ramificação de uma nova variável fracionária selecionada, e o problema original é então reotimizado a partir de um novo nó da árvore de busca. O processo é finalizado após um limite de tempo estabelecido ou ao término da exploração dos nós gerados pela árvore $B \mathscr{E} B$.

Devida à complexidade combinatorial das RES o algoritmo $B \mathscr{E} C$ muitas vezes não é capaz de encontrar soluções incumbentes, especialmente para problemas de médio e grande porte. Para contornar essa limitação, propomos uma estratégia para a obtenção de uma solução factível inicial, partindo de uma reformulação do modelo da seção 2 que desconsidera as variáveis de rota $y_{u v}^{k j t}$. A nova função objetivo $\left(F O^{\prime}\right)$, dada por 24 considera o custo de entrega direta, caso o cliente seja servido pelo veículo $k$ designado à planta $j$ no período $t$. 


$$
\min \sum_{t \in \mathcal{T} \cup\{p+1\}} \sum_{l \in \mathcal{C}} h_{l} I_{l}^{t}+\sum_{j \in \mathcal{P}} \sum_{l \in \mathcal{C}} \sum_{k \in \mathcal{K}} \sum_{t \in \mathcal{T}} c_{j l} Y_{j l}^{k t}
$$

$\mathrm{A}\left(F O^{\prime}\right)$ está sujeito às restrições $(2)-(7) \cup \sqrt{10}-\sqrt{12}$, resultando em um modelo de fluxo com entregas diretas. A política $O U$ é igualmente contemplada adicionando as restrições $(15)-(17)$ ao modelo de fluxo.

Para gerar as rotas iniciais e completar a solução incumbente do MDIRPSD, resolvemos os Travel Salesman Problem (TSP's), para as triplas $(j, k, t)$ associadas às variáveis $Y_{j l}^{k t}=$ 1, pelo método proposto por [13]. A solução inicial obtida é então inserida no nó raiz do $B \mathscr{E} C$ proposto. Um esquema para o algoritmo $B \mathscr{E} C$ é apresentado em 1 .

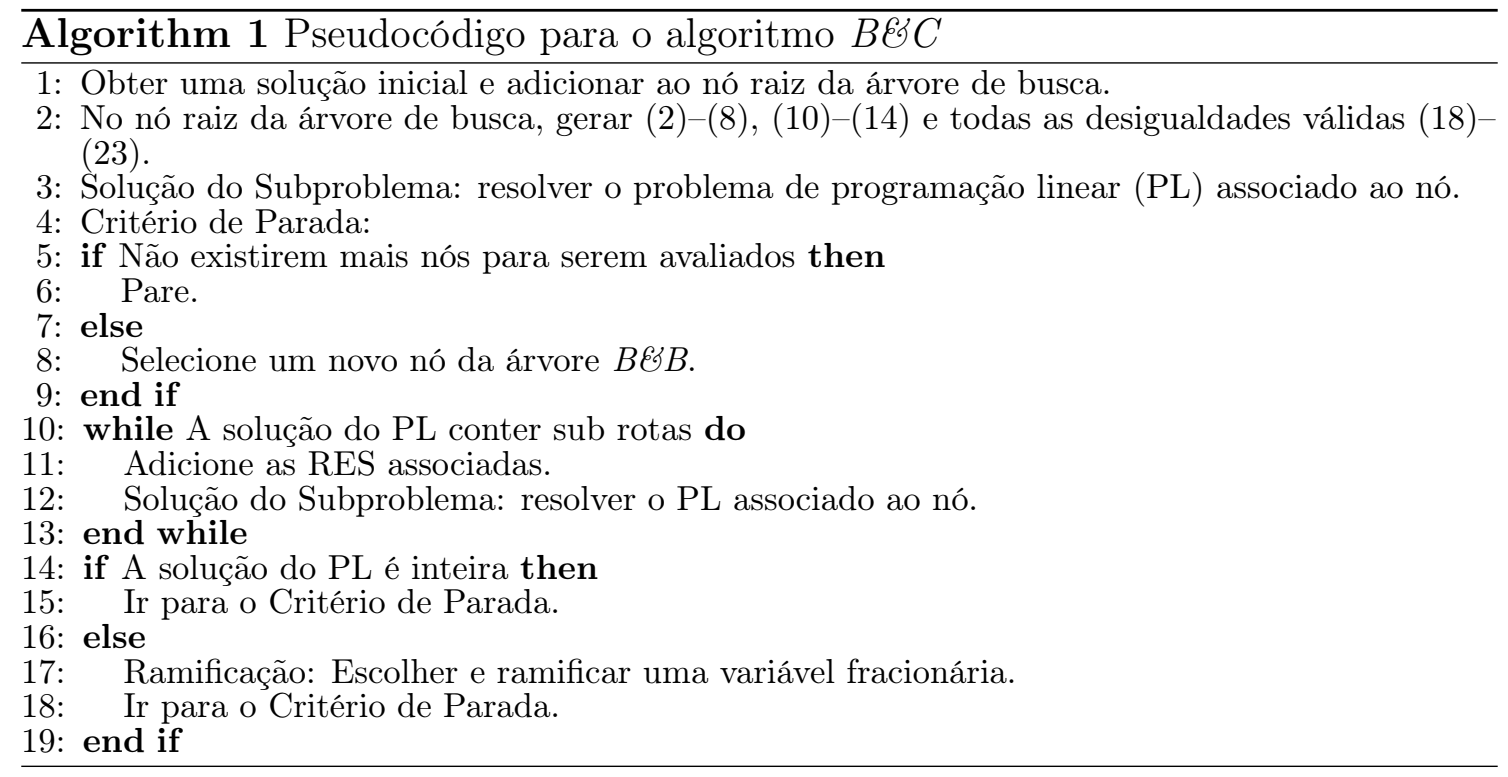

\section{EXPERIMENTOS COMPUTACIONAIS}

Os testes computacionais foram realizados em processadores Core i7, com 16 GB de memória RAM e sistema operacional Linux 6.6. O algoritmo $B \& C$, descrito na seção 3 . foi implementado em $C++$ e os modelos de programação linear inteira foram resolvidos com o solver Gurobi 8.1 .0 com parâmetros default. O algoritmo BEC foi processado por até 7200 segundos.

O $B \mathscr{E} C$ foi testado sobre um conjunto de 20 instâncias, adaptadas de [10] para múltiplos depósitos por [5]. Todas as instâncias possuem frota com 3 veículos e o número de plantas depende do número de clientes. Da esquerda para a direita, a Tabela 1 reporta o nome da instância na seguinte estrutura $\ln |\mathcal{C}| \mathrm{h}|\mathcal{T}|$, onde $|\mathcal{C}|$ é o número de clientes e $|\mathcal{T}|$ o número de períodos. Sequencialmente, são apresentados as soluções (Upper Bounds - UB) obtidos pelo $B \mathscr{E} C$ proposto por 5] com o tempo de processamento na coluna ao lado. As quatro colunas seguintes apresentam os resultados do nosso $B \mathscr{E} C$, envolvendo o $U B$, (Lower Bounds - LB), o desvio entre eles e o tempo de processamento. A última coluna informa o desvio entre os $U B$ 's, calculado por $G A P=\frac{U B_{\text {nosso }}-U B_{\text {bertazzi }}}{U B_{\text {bertazzi }}}$, quantificando a comparação entre os métodos. Pela dificuldade inerente ao problema, foi possível encontrar soluções ótimas em apenas 3 instâncias, limitadas à 10 clientes $(1 n 5 h 3,1 n 10 h 3$ e $1 n 5 h 6)$, enquanto instâncias com 50 clientes apresentam gaps superiores à $40 \%$. Contudo, esse padrão de desempenho é esperado para métodos exatos em problemas dessa natureza 3. 


\begin{tabular}{c|cc|cccc|c}
\hline \multirow{2}{*}{ Instancia } & \multicolumn{1}{|c|}{ Bertazzi el al. (2019) } & \multicolumn{4}{|c|}{ B\&C } & \multirow{2}{*}{ GAP } \\
\cline { 2 - 6 } & UB & T(s) & UB & LB & $\frac{U B-L B}{U B}$ & T(s) & \\
\hline 1n5h3 & 1148,80 & 89,71 & 1148,80 & 1148,80 & $0,00 \%$ & 1,11 & $0,00 \%$ \\
1n10h3 & 2177,99 & 21700 & 2110,01 & 2109,98 & $0,00 \%$ & 664,70 & $-3,12 \%$ \\
1n15h3 & 4588,88 & 21700 & 4013,73 & 3768,66 & $6,11 \%$ & 7200 & $-12,53 \%$ \\
1n20h3 & 3263,25 & 21700 & 3110,41 & 2862,78 & $7,96 \%$ & 7200 & $-4,68 \%$ \\
1n25h3 & 4203,45 & 21700 & 3548,92 & 3183,22 & $10,30 \%$ & 7200 & $-15,57 \%$ \\
1n30h3 & 5206,49 & 21700 & 4342,43 & 3706,57 & $14,64 \%$ & 7200 & $-16,60 \%$ \\
1n35h3 & 7013,75 & 21700 & 5699,71 & 4682,80 & $17,84 \%$ & 7200 & $-18,74 \%$ \\
1n40h3 & 7080,27 & 21700 & 5908,26 & 4702,24 & $20,41 \%$ & 7200 & $-16,55 \%$ \\
1n45h3 & 7272,02 & 21700 & 7088,22 & 4591,92 & $35,22 \%$ & 7200 & $-2,53 \%$ \\
1n50h3 & 7881,37 & 21700 & 7874,59 & 5077,56 & $35,52 \%$ & 7200 & $-0,09 \%$ \\
1n5h6 & 2595,14 & 8685,15 & 2595,14 & 2595,14 & $0,00 \%$ & 3,37 & $0,00 \%$ \\
1n10h6 & 5102,45 & 21700 & 4214,91 & 3644,21 & $13,54 \%$ & 7200 & $-17,39 \%$ \\
1n15h6 & 9904,03 & 21700 & 8056,82 & 7376,30 & $8,45 \%$ & 7200 & $-18,65 \%$ \\
1n20h6 & 11433,40 & 21700 & 10519,36 & 8501,48 & $19,18 \%$ & 7200 & $-7,99 \%$ \\
1n25h6 & 11949,99 & 21700 & 11484,49 & 8732,21 & $23,97 \%$ & 7200 & $-3,90 \%$ \\
1n30h6 & 12750,90 & 21700 & 12511,56 & 8670,60 & $30,70 \%$ & 7200 & $-1,88 \%$ \\
1n35h6 & 12924,00 & 21700 & 11347,92 & 8897,96 & $21,59 \%$ & 7200 & $-12,19 \%$ \\
1n40h6 & 12208,50 & 21700 & 13010,85 & 9992,68 & $23,20 \%$ & 7200 & $6,57 \%$ \\
1n45h6 & 10884,90 & 21700 & 11019,43 & 7490,82 & $32,02 \%$ & 7200 & $1,24 \%$ \\
1n50h6 & 14031,90 & 21700 & 14081,32 & 8260,21 & $41,34 \%$ & 7200 & $0,35 \%$ \\
\hline Média & $\mathbf{7 6 8 1 , 0 7}$ & $\mathbf{1 9 9 6 8 , 7 4}$ & $\mathbf{7 1 8 4 , 3 4}$ & $\mathbf{5 4 9 9 , 8 1}$ & $\mathbf{1 8 , 1 0 \%}$ & $\mathbf{6 1 5 5 , 0 5}$ & $\mathbf{- 7 , 2 1 \%}$ \\
\hline
\end{tabular}

Tabela 1: Resultados comparativos com o $B \mathscr{E} C$ proposto por [5]

A análise comparativa entre algoritmos apresenta dados interessantes. Salientamos que os resultados de 5 não reportam os Lower Bounds $(L B)$ e o que o tempo de processamento foi de 21000 segundos, muito superior aos 7200 que adotamos. Embora a diferença de implementação, solver e hardware não permitam comparações justas, podemos observar em 17 das 20 instâncias o $U B$ obtido pelo nosso algoritmo foi melhor que o UB dos autores comparados. Além disso, conseguimos provar a otimalidade para a instância $1 n 10 h 3 \mathrm{em}$ 664,7 segundos, ao passo que os [5] não o fez em 21000 segundos. A diferença no tempo computacional pode ser explicada pela solução inicial fornecida, o que pode direcionar o esforço do método para provar a otimalidade, em detrimento ao processo de obtenção de uma solução factível. De forma geral, conseguimos reduzir os custos em 7,21\% em média.

O segundo grupo de análise compara as políticas de estoque $M L$ e $O U$ sem considerar os custos de manutenção, ou seja, $h_{l}=0, l \in \mathcal{C}$. Da esquerda para a direita a Tabela 2 apresenta o nome das instâncias, os resultados da política $M L$ para $U B, L B$, desvio e tempo de processamento em segundos, seguido das mesmas informações para a política $O U$. A última coluna compara os $U B$ 's entre as políticas, calculada por $G A P=\frac{U B_{O U}-U B_{M L}}{U B_{M L}}$. A dificuldade na resolução das políticas foi semelhante no âmbito do tempo de processamento. Em termos de solução, o $B \mathscr{E} C$ para a política $O U$ encontrou uma solução ótima a mais que a política $M L$ (instância $1 n 15 h 3$ ), devido à redução do número de visitas. O desempenho geral do algoritmo $B E \mathcal{E} C$ foi semelhante entre as políticas, com tempo médio global e desvio médio entre $U B$ e $L B$ muito próximos. Já pelo aspecto do custo total, a política $O U$ é em média $6,47 \%$ mais custosa, mesmo sem considerar o custo de estocagem nos clientes. Esse padrão de resposta é condizente com a formulação do problema, pois, em último caso, a política $M L$ recai na política $O U$.

Por fim, comparamos as duas políticas para a formulação apresentada na seção 2 . 


\begin{tabular}{c|cccc|cccc|c}
\hline \multirow{2}{*}{ Instância } & \multicolumn{7}{|c|}{ Política ML } & \multicolumn{4}{c|}{ Política OU } & \multirow{2}{*}{ GAP } \\
\cline { 2 - 8 } & UB & LB & $\frac{U B-L B}{U B}$ & T(s) & UB & LB & $\frac{U B-L B}{U B}$ & T(s) & \\
\hline 1n5h3 & 1148,80 & 1148,80 & $0,00 \%$ & 1,11 & 1177,69 & 1177,69 & $0,00 \%$ & 0,51 & $2,51 \%$ \\
1n10h3 & 2110,01 & 2109,98 & $0,00 \%$ & 664,70 & 2298,24 & 2298,24 & $0,00 \%$ & 646,03 & $8,92 \%$ \\
1n15h3 & 4013,73 & 3768,66 & $6,11 \%$ & 7200 & 4173,13 & 4173,44 & $0,01 \%$ & 7112,54 & $3,98 \%$ \\
1n20h3 & 3110,41 & 2862,78 & $7,96 \%$ & 7200 & 3313,31 & 3121,11 & $5,80 \%$ & 7200 & $6,52 \%$ \\
1n25h3 & 3548,92 & 3183,22 & $10,30 \%$ & 7200 & 3677,37 & 3595,77 & $2,22 \%$ & 7200 & $3,62 \%$ \\
1n30h3 & 4342,43 & 3706,57 & $14,64 \%$ & 7200 & 4397,71 & 4082,52 & $7,17 \%$ & 7200 & $1,27 \%$ \\
1n35h3 & 5699,71 & 4682,80 & $17,84 \%$ & 7200 & 7340,37 & 5155,16 & $29,77 \%$ & 7200 & $28,79 \%$ \\
1n40h3 & 5908,26 & 4702,24 & $20,41 \%$ & 7200 & 7429,49 & 5126,49 & $31,00 \%$ & 7200 & $25,75 \%$ \\
1n45h3 & 7088,22 & 4591,92 & $35,22 \%$ & 7200 & 7351,31 & 4709,15 & $35,94 \%$ & 7200 & $3,71 \%$ \\
1n50h3 & 7874,59 & 5077,56 & $35,52 \%$ & 7200 & 8622,25 & 5216,66 & $39,50 \%$ & 7200 & $9,49 \%$ \\
1n5h6 & 2595,14 & 2595,14 & $0,00 \%$ & 3,37 & 2607,74 & 2607,74 & $0,00 \%$ & 2,83 & $0,49 \%$ \\
1n10h6 & 4214,91 & 3644,21 & $13,54 \%$ & 7200 & 4497,83 & 3994,43 & $11,19 \%$ & 7200 & $6,71 \%$ \\
1n15h6 & 8056,82 & 7376,30 & $8,45 \%$ & 7200 & 8438,64 & 7474,66 & $11,42 \%$ & 7200 & $4,74 \%$ \\
1n20h6 & 10519,36 & 8501,48 & $19,18 \%$ & 7200 & 12312,43 & 8940,64 & $27,39 \%$ & 7200 & $17,05 \%$ \\
1n25h6 & 11484,49 & 8732,21 & $23,97 \%$ & 7200 & 11099,11 & 8749,27 & $21,17 \%$ & 7200 & $-3,36 \%$ \\
1n30h6 & 12511,56 & 8670,60 & $30,70 \%$ & 7200 & 13153,27 & 8450,91 & $35,75 \%$ & 7200 & $5,13 \%$ \\
1n35h6 & 11347,92 & 8897,96 & $21,59 \%$ & 7200 & 11295,75 & 8541,92 & $24,38 \%$ & 7200 & $-0,46 \%$ \\
1n40h6 & 13010,85 & 9992,68 & $23,20 \%$ & 7200 & 13348,46 & 10293,58 & $22,89 \%$ & 7200 & $2,59 \%$ \\
1n45h6 & 11019,43 & 7490,82 & $32,02 \%$ & 7200 & 11221,44 & 8489,99 & $24,34 \%$ & 7200 & $1,83 \%$ \\
1n50h6 & 14081,32 & 8260,21 & $41,34 \%$ & 7200 & 14086,57 & 8750,18 & $37,88 \%$ & 7200 & $0,04 \%$ \\
\hline Média & $\mathbf{7 1 8 4 , 3 4}$ & $\mathbf{5 4 9 9 , 8 1}$ & $\mathbf{1 8 , 1 0 \%}$ & $\mathbf{5 7 4 7 , 4 6}$ & $\mathbf{7 5 9 2 , 1 2}$ & $\mathbf{6 1 5 5 , 0 5}$ & $\mathbf{1 8 , 3 9 \%}$ & $\mathbf{6 1 4 9 , 8 6}$ & $\mathbf{6 , 4 7 \%}$ \\
\hline
\end{tabular}

Tabela 2: Resultados comparativos para as políticas $O U$ e $M L$ sem considerar os custos de estocagem

considerando os custos de estocagem nos clientes. A Tabela 3 reporta a mesma estrutura da tabela anterior. Com relação ao desempenho do algoritmo $B \mathscr{G} C$, verificamos que as soluções ótimas encontradas são as mesmas para as duas políticas, com desvios globais médios semelhantes para o tempo de processamento e os resultados dos limitantes $U B$ e LB. Já a análise comparativa destaca a importância dos custos de estocagem. Como a política $O U$ mantém níveis de estoque mais elevados nos clientes, a comparação entre as políticas é mais díspar. Em valores médios para o conjunto das 20 instâncias testadas, a política $O U$ é $11,95 \%$ mais custosa que a política $M L$. Esse dado é condizente com os resultados reportados por [10, 14, 4], que resolvem diferentes variações do Inventory Routing Problem sob a política de estoque $O U$. 


\begin{tabular}{c|cccc|cccc|c}
\hline \multirow{2}{*}{ Instância } & \multicolumn{7}{|c|}{ Política ML } & \multicolumn{4}{c|}{ Política OU } & \multirow{2}{*}{ GAP } \\
\cline { 2 - 8 } & UB & LB & $\frac{U B-L B}{U B}$ & T(s) & UB & LB & $\frac{U B-L B}{U B}$ & T(s) & \\
\hline 1n5h3 & 1278,11 & 1278,11 & $0,00 \%$ & 0,74 & 1378,28 & 1378,28 & $0,00 \%$ & 0,44 & $7,84 \%$ \\
1n10h3 & 2671,71 & 2671,71 & $0,00 \%$ & 466,45 & 3050,06 & 3050,06 & $0,00 \%$ & 198,89 & $14,16 \%$ \\
1n15h3 & 4588,73 & 4501,15 & $1,91 \%$ & 7200 & 5035,57 & 5035,57 & $0,00 \%$ & 6019,75 & $9,74 \%$ \\
1n20h3 & 3938,59 & 3832,00 & $2,71 \%$ & 7200 & 4513,63 & 4300,28 & $4,73 \%$ & 7200 & $14,60 \%$ \\
1n25h3 & 4512,84 & 4200,14 & $6,93 \%$ & 7200 & 5035,51 & 4882,98 & $3,03 \%$ & 7200 & $11,58 \%$ \\
1n30h3 & 5756,19 & 5435,67 & $5,57 \%$ & 7200 & 6726,83 & 6394,82 & $4,94 \%$ & 7200 & $16,86 \%$ \\
1n35h3 & 7008,24 & 6204,91 & $11,46 \%$ & 7200 & 8573,82 & 7146,71 & $16,64 \%$ & 7200 & $22,34 \%$ \\
1n40h3 & 7969,30 & 6748,08 & $15,32 \%$ & 7200 & 9835,01 & 7838,64 & $20,30 \%$ & 7200 & $23,41 \%$ \\
1n45h3 & 9083,14 & 6669,32 & $26,57 \%$ & 7200 & 9942,74 & 7412,93 & $25,44 \%$ & 7200 & $9,46 \%$ \\
1n50h3 & 10282,09 & 7311,93 & $28,89 \%$ & 7200 & 11513,42 & 7876,56 & $31,59 \%$ & 7200 & $11,98 \%$ \\
1n5h6 & 3023,43 & 3023,43 & $0,00 \%$ & 3,02 & 3238,46 & 3238,46 & $0,00 \%$ & 2,17 & $7,11 \%$ \\
1n10h6 & 5111,14 & 4497,20 & $12,01 \%$ & 7200 & 5576,66 & 5162,65 & $7,42 \%$ & 7200 & $9,11 \%$ \\
1n15h6 & 9267,71 & 8472,42 & $8,58 \%$ & 7200 & 9880,86 & 8995,69 & $8,96 \%$ & 7200 & $6,62 \%$ \\
1n20h6 & 11860,02 & 9944,18 & $16,15 \%$ & 7200 & 14953,22 & 10947,82 & $26,79 \%$ & 7200 & $26,08 \%$ \\
1n25h6 & 11704,31 & 10114,42 & $13,58 \%$ & 7200 & 14016,66 & 10675,82 & $23,83 \%$ & 7200 & $19,76 \%$ \\
1n30h6 & 15528,29 & 11331,69 & $27,03 \%$ & 7200 & 16942,74 & 12055,63 & $28,84 \%$ & 7200 & $9,11 \%$ \\
1n35h6 & 13799,01 & 11140,03 & $19,27 \%$ & 7200 & 14285,10 & 11417,87 & $20,07 \%$ & 7200 & $3,52 \%$ \\
1n40h6 & 16967,71 & 13221,60 & $22,08 \%$ & 7200 & 17981,20 & 14346,50 & $20,21 \%$ & 7200 & $5,97 \%$ \\
1n45h6 & 14660,56 & 11175,57 & $23,77 \%$ & 7200 & 15690,00 & 12538,76 & $20,08 \%$ & 7200 & $7,02 \%$ \\
1n50h6 & 18676,15 & 11302,30 & $39,48 \%$ & 7200 & 19167,84 & 12909,04 & $32,65 \%$ & 7200 & $2,63 \%$ \\
\hline Média & $\mathbf{8 8 8 4 , 3 6}$ & $\mathbf{7 1 5 3 , 7 9}$ & $\mathbf{1 4 , 0 7 \%}$ & $\mathbf{6 1 4 3 , 5 1}$ & $\mathbf{9 8 6 6 , 8 8}$ & $\mathbf{7 8 8 0 , 2 5}$ & $\mathbf{1 4 , 7 8 \%}$ & $\mathbf{6 0 7 1 , 0 6}$ & $\mathbf{1 1 , 9 5 \%}$ \\
\hline
\end{tabular}

Tabela 3: Resultados comparativos para as políticas $O U$ e $M L$ considerando custos de estocagem

\section{CONCLUSÕES}

Neste trabalho abordamos um problema logístico prático, que emerge do paradigma do estoque gerenciado pelo fornecedor um em logística urbana, denominado Multi Depot Inventory Routing Problem with Split Deliveries (MDIRPSD) ou Problema de Roteirização e Estoques com Múltiplos Depósitos e Entregas Fracionadas. A partir do trabalho de [5] que introduziu o problema na literatura, nós expandimos a formulação e incorporamos os custos de estocagem no cliente. Além disso, adaptamos a política de estoque $O U$ para o $M D I R P S D$ e desenvolvemos um novo algoritmo $B \mathscr{G} C$ com um mecanismo de obtenção de uma solução inicial, além de algumas alterações estruturais. Os resultados computacionais apontam que nosso método é superior à abordagem proposta por [5], sendo capaz de reduzir os UB's conhecidos, além de provar a otimalidade para uma instância em aberta. A análise comparativa entre as políticas de estoque $M L$ e $O U$ demonstram que níveis mais elevados de estoque geram soluções mais custosas, e destacam a importância de se considerar os custos de estoque no processo decisório.

\section{AGRADECIMENTOS}

Agradecemos ao Centro de Computação Científica e Software Livre (C3SL) da Universidade Federal do Paraná pela assistência e recursos computacionais oferecidos. Agradecemos à Coordenação de Aperfeiçoamento de Pessoal de Nível Superior (CAPES), pelo apoio financeiro N. 1554767. 


\section{REFERÊNCIAS BIBLIOGRÁFICAS}

[1] ANDERSSON, H. et al. Industrial aspects and literature survey: Combined inventory management and routing. v. 37, n. 9, p. 1515-1536, 2010. 2

[2] GOVINDAN, K. Vendor-managed inventory: A review based on dimensions. International Journal of Production Research, v. 51, n. 13, p. 3808-3835, 2013. 2

[3] COELHO, L. C.; CORDEAU, J.-F.; LAPORTE, G. Thirty Years of Inventory Routing. Transportation Science, v. 48, n. 1, p. 1-19, 2013. 2, 3, 7

[4] GUIMARÃES, T. A. et al. The two-echelon multi-depot inventory-routing problem. Computers and Operations Research, v. 101, p. 220-233, 2019. 2, 6, 9

[5] BERTAZZI, L. et al. A matheuristic algorithm for the multi-depot inventory routing problem. Transportation Research Part E: Logistics and Transportation Review, v. 122, p. 524-544, 2019. 2, 3, 5, 7, 8, 10

[6] COELHO, L. C.; LAPORTE, G. A branch-and-cut algorithm for the multi-product multi-vehicle inventory-routing problem. International Journal of Production Research, v. 51, n. $23-24$, p. $7156-7169,2013.2$

[7] COELHO, L. C.; LAPORTE, G. The exact solution of several classes of inventoryrouting problems. Computers and Operations Research, v. 40, n. 2, p. 558-565, 2013. 2

[8] SERnA, M. D. A.; CORTES, J. A. Z.; SEPulvedA, D. G. Modeling the Inventory Routing Problem (IRP) with multiple depots with genetic algorithms. IEEE Latin America Transactions, v. 13, n. 12, p. 3959-3965, 2015. 2

[9] BeRTAZZI, L.; PALETTA, G.; SPERANZA, M. G. Deterministic Order-Up-To Level Policies in an Inventory Routing Problem. Transportation Science, v. 36, n. 1, p. 119132, 2003. 3

[10] ARCHETTI, C. et al. A branch-and-cut algorithm for a vendor-managed inventoryrouting problem. Transportation Science, v. 41, n. 3, p. 382-391, 2007. 5, 7, 9

[11] COELHO, L. C.; LAPORTE, G. Improved solutions for inventory-routing problems through valid inequalities and input ordering. International Journal of Production Economics, Elsevier, v. 155, p. 391-397, 2014. 5

[12] LYSGAARD, J.; LETCHFORD, A. N.; EGLESE, R. W. A new branch-and-cut algorithm for the capacitated vehicle routing problem. Mathematical Programming, v. 100, n. 2 , p. $423-445,2004$. 6

[13] PADBERG, M.; RINALDI, G. A Branch-and-Cut Algorithm for the Resolution of Large-Scale Symmetric Traveling Salesman Problems. SIAM Review, v. 33, n. 1, p. 60 100, 1991. 7

[14] COelho, L. C.; CORdeaU, J. F.; LAPORTE, G. Consistency in multi-vehicle inventory-routing. Transportation Research Part C: Emerging Technologies, v. 24, p. 270-287, 2012. 9 